\title{
Factores determinantes de éxito de la rehabilitación cardiovascular en pacientes coronarios sometidos a revascularización miocárdica
}

\author{
Claudio Chamorro ${ }^{1,2,{ }^{*}}$; Dominique Guidi ${ }^{1}$; Fernando Yáñez ${ }^{3}$; Gastón Chamorro $^{3}$ \\ 1 Carrera de Kinesiología, UDA, Cs de la Salud, Facultad de Medicina, Pontificia Universidad Católica de Chile, Santiago, Chile; \\ 2 Servicio Kinesiología, Clínica UC San Carlos de Apoquindo, Santiago, Chile; 3 División de Enfermedades Cardiovasculares, \\ Facultad de Medicina, Pontificia Universidad Católica de Chile. \\ Los autores no declaran conflictos de interés ni fuentes de apoyo
}

Introducción: La rehabilitación cardiovascular ha demostrado tener efectos beneficiosos en pacientes con antecedentes de patología coronaria.

Objetivos: Identificar los factores que determinan el resultado de un programa de rehabilitación cardiovascular (PRC) aplicado a pacientes coronarios revascularizados.

Métodos: 67 pacientes sometidos a cirugía de bypass o angioplastia fueron evaluados en su capacidad funcional mediante el test de marcha de 6 minutos (TM) al inicio y al completar el programa de rehabilitación cardiovascular. La distancia recorrida en el test de marcha fue correlacionada con la edad, capacidad funcional previa al PRC, tiempo en completar el programa, tiempo que media entre la intervención y el inicio del programa, duración del programa y tipo de revascularización. Además, se comparó el incremento de la capacidad funcional entre los pacientes que fueron derivados a 36 sesiones con los referidos a solo 12 . Resultados: 67 pacientes cumplieron los criterios para evaluación del PRC. Globalmente, se observó una mejoría de $12 \%$ (511,4 a 573,4 m) en la distancia del TM $(\mathrm{p}<0.001))$. El mayor beneficio, en términos de distancia en el TM se obtuvo al efectuar un programa con más sesiones ( 36 vs 12 ) con valores de $20 \%$ y $8 \%$, respectivamente $(\mathrm{p}<0.002)$. El poder terminar el PRC de 36 sesiones más rápidamente (entre 10 y 13 semanas vs entre 14 y 24 semanas se asoció a una mayor incremente en el TM con valores de $19 \%$ vs $10 \%$, respectivamente $(\mathrm{p}<0,003)$. El incremento en el TM no difirió entre 3 grupos de edad (desde 49 a 85 años); en el tiempo que transcurre entre la intervención y el inicio del PRC (antes vs después de la 8a semana post revascularización), al tipo de revascularización a la que fue sometido el paciente (cirugía o angioplastía) y a la capacidad funcional previa que estos presentan al inicio del PRC.

Conclusión: El PRC es efectivo en mejorar significativamente la capacidad funcional de pacientes revascularizados, especialmente cuando el número de sesiones del programa es mayor y cuando se realiza con una frecuencia de al menos 3 veces por semana. El PRC es igualmente efectivo en pacientes enviados a rehabilitación en forma más precoz, como también lo es en sujetos más añosos. Estos efectos fueron independientes del tipo de revascularización. 


\section{Cardiovascular Rehabilitation in patients following myocardial revascularization: factors associated to program success}

Introduction: Background: Cardiovascular Rehabilitation Programs (CRP) have been shown to produce beneficial effects in patients with coronary artery disease. Aim: to identify factors associated to CRP success in patients who underwent myocardial revascularization

Methods: 67 patients who underwent coronary artery bypass surgery $(\mathrm{CABG})$ or percutaneous coronary artery angioplasty (PTCA) were evaluated for functional capacity by means of a standard 6 min walking test ( $6 \mathrm{mWT})$, before and after completion of the CRP. Distance covered during the test was correlated with age, prior functional class, time employed to complete CRP, time from coronary intervention and CRP initiation, CRP duration and type of revascularization. In addition, patients referred for a 36 sessions CRP were compared to those referred to only 12 sessions.

Results: 67 patients met inclusion criteria. Overa11 , there was a $12 \%$ increase $(511,4$ to $573,4 \mathrm{~m})$ in $6 \mathrm{mWT}$ distance $(\mathrm{p}<0.001)$. The greatest benefit was obtained with the 36 session CRP as compared to a 12 session CRP (20 vs $8 \%(\mathrm{p}<0.002)$. Also, completion of a 36 session CRP between 10 and 13 weeks compared to 14 to 24 weeks revealed a greater benefit in the former group (19\% vs $10 \%$, respectively $(\mathrm{p}<0.003)$. There was no difference in 6 minWT distance in 3 groups of age (extending from 49 to 85 years-old); In addition, time from intervention to initiation of CRP (before vs after 8 weeks), type of revascularization or functional capacity at the beginning of CRP showed any difference in 6mWT distance.

Conclusion: CRP is a highly effective intervention to improve functional capacity in patients following myocardial revascularization, more so when more sessions are employed and when at least 3 sessions per week are implemented. The program is equally effective in patients starting CRP early after revascularization, and benefit is independent from patient age.

Keywords: cardiac rehabilitation; myocardial revascularization; coronary artery bypass surgery, angioplasty, tansluminal, coronary 


\section{Introducción:}

La rehabilitación cardiovascular se define como un conjunto de interacciones coordinadas que busca optimizar el funcionamiento físico, psíquico y social del paciente con patología cardiovascular ${ }^{1-4}$. El programa debe incluir, aparte del ejercicio físico, apoyo psicológico, nutricional, terapia farmacológica y educación sobre factores de riesgo ${ }^{4}$. Sin embargo, programas internacionales siguen considerando al ejercicio físico como el elemento central de la rehabilitación cardiovascular $2,6,7$.

La principal relevancia de los programas de rehabilitación cardiovascular basados en el ejercicio radica en la disminución en la mortalidad y morbilidad en los pacientes con presencia de eventos cardiovasculares. Existe suficiente evidencia sobre los factores centrales y periféricos que llevan a intolerancia al ejercicio físico en pacientes con cardiopatía isquémica y con insuficiencia cardíaca ${ }^{8-11}$. Se sabe que muchos de estos factores pueden mejorar con un buen programa de rehabilitación basado en el ejercicio, logrando una mejoría en la capacidad funcional y en la actividad de la vida diaria del paciente cardiópata ${ }^{2,12}$.

Existen escasas publicaciones metodológicamente confiables sobre el efecto del ejercicio en pacientes con patología coronaria. Las principales razones de ello son la dificultad de obtener una muestra suficientemente grande para que sea confiable, la inclusión de diferentes patologías y diversos protocolos dentro de una misma muestra y la escasa adherencia para completar el programa de rehabilitación que, como promedio recomendado por la American Heart Association, dura 3 a 4 meses. Es común encontrar sobreestimaciones del beneficio del ejercicio en pacientes cardiópatas ${ }^{13}$. Probablemente, por todas estas razones, el PRC está siendo subutilizado en países desarrollados y, sobre todo, en países en vías de desarro1lo. De hecho, en Estados Unidos sólo entre 10 y $20 \%$ de los potenciales candidatos participan en este programa ${ }^{14}$. Existe una gran variabilidad en los beneficios que produce el ejercicio en distintos sujetos coronarios incluidos en el programa de rehabilitación incluyendo mejorías en la capacidad funcional y reducción de la mortalidad cardiovascular ${ }^{15}$. Diversas publicaciones han demostrado mejorías entre el 10 y $40 \%$ en el consumo máximo de oxígeno (VO2 máx.) en distintos sujetos sometidos al mismo programa de rehabilitación ${ }^{16-18}$.

Sin embargo, no existe claridad ni consenso sobre: a) qué tipo de pacientes se benefician más con el ejercicio; b) cuándo es recomendable que los pacientes revascularizados inicien el programa de rehabilitación y c) cómo debe

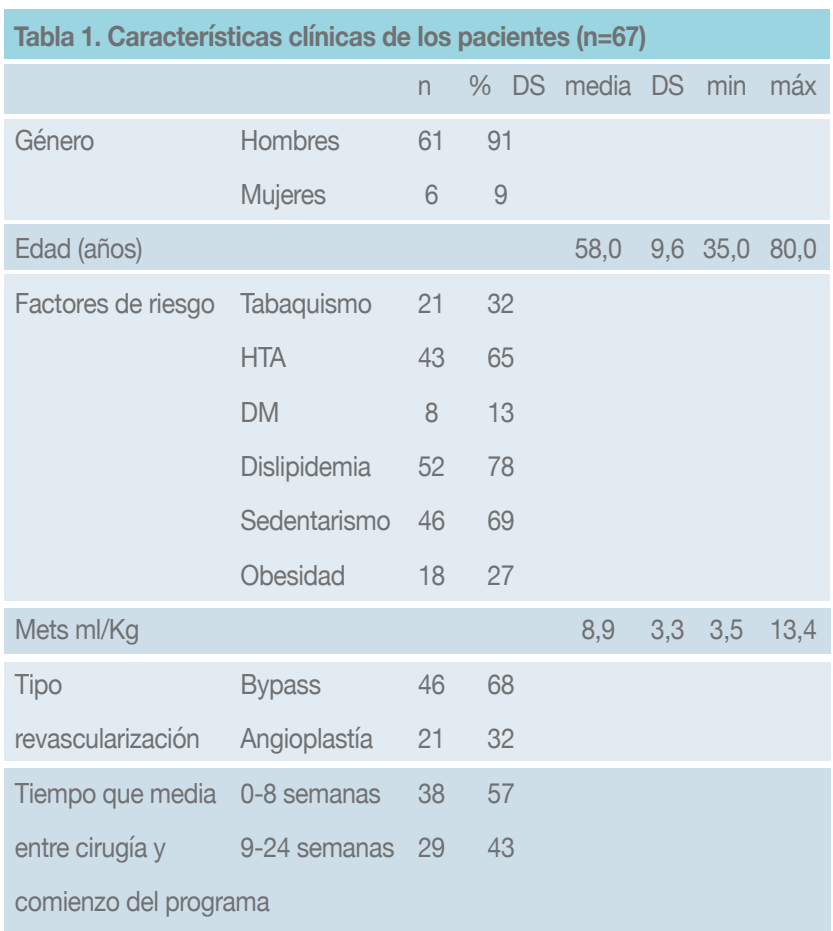

HTA: hipertensión arterial; DM: diabetes mellitus; Mets: unidad de medida del índice metabólico (cantidad de energía que consume un individuo en situación de reposo en un minuto) y corresponde a $3,5 \mathrm{ml} \mathrm{O2/kg}$

desarrollarse el programa para obtener mayores logros. La presente investigación busca determinar cuáles son los principales factores que determinan el beneficio del ejercicio en pacientes con cardiopatía isquémica sometidos a cirugía de revascularización o angioplastía. Interesa conocer el efecto de la edad, capacidad funcional del sujeto y tiempo que transcurre entre la operación y el inicio del programa.

\section{Material y Método:}

\subsection{Pacientes}

Se reclutaron 67 pacientes (6 mujeres y 61 hombres) que participan en el programa de rehabilitación cardiovascular (PRC) del Centro Médico San Jorge de la Facultad de medicina de la Universidad Católica entre abril de 2005 y diciembre de 2015. Los criterios de inclusión fueron: i) pacientes con angioplastía transluminal de arterias coronarias (PTCA) o bypass aortocoronario (PAC); ii) revascularización dentro de los 6 meses previos al inicio del programa de rehabilitación. iii) edad $>30$ y $<85$ años; iv) test de esfuerzo de no más de 6 meses de antigüedad. Los criterios de exclusión fueron : i) incapacidad física para efectuar la rehabilitación, de origen neurológico, traumatológico o psicológico. ii) presencia de comorbilidad que 
afecte el pronóstico de vida del paciente. iii) presencia de patología agregada que altere el programa de rehabilitación. iv) no completar las 36 sesiones de rehabilitación dentro de los 5 meses post inicio del programa. v) ser sometido tanto a PAC como PTCA. Todos los participantes firmaron consentimiento informado de acuerdo a la declaración de Helsinski. Las características de los sujetos se presentan en la Tabla 1.

\subsection{Evaluación de la capacidad funcional mediante el test de marcha}

Este test tiene una correlación Inter evaluador entre 0,86 y 0,95 y se correlaciona bastante bien con el VO2 max $(r=0,52-0,71)$. Se ubicaron 2 conos a una distancia de 25 metros y se le pidió al paciente que caminara lo más rápido posible durante 6 minutos cubriendo la distancia entre los conos. Al completar cada minuto se motivó al paciente y se le señalaron los minutos restantes. Si lo necesitare, el paciente pudo detenerse y continuar. Presencia de angina, dolor en las piernas, mareos y presión sobre 180/100 fueron causales de suspensión del test. Se midió frecuencia cardíaca, presión arterial previa y posterior a la prueba y se le consultó sobre la sensación subjetiva de fatiga. En todo momento, al menos un pie estuvo en contacto con el suelo.

\subsection{Evaluación de ingreso}

Al paciente se le registraron datos personales, diagnóstico, fecha operación, fecha de inicio del programa, fármacos en uso y capacidad funcional expresada en METs, evaluada en el test de esfuerzo exigido previo al programa. Luego se realizó una evaluación musculoesquelética por el kinesiólogo, para determinar si presentaba patologías agregadas que ameritasen alguna variación en el programa de rehabilitación. A continuación, se obtuvo el $\mathrm{R}$ máximo, que es la máxima carga que el paciente puede levantar una vez en las siguientes máquinas de fuerza: press pierna, isquiotibiales, mesa de cuádriceps, press banca y pull down. Finalmente, se efectuó el test de marcha de 6 minutos. Estas mediciones se repitieron cuando el paciente completó la última sesión.

Según la edad, los pacientes fueron clasificados en grupos de edad 40 a 59 años; 60 a 74 años y 75 a 85 años. Según la capacidad funcional determinada por un test de esfuerzo al inicio del programa, los pacientes se clasificaron en < 5 Mets; 5-8 Mets y sobre 8 Mets. Según el número de sesiones del programa de rehabilitación se clasificaron en pacientes que realizan 12 sesiones y aquellos que realizan 36 sesiones. Según el tiempo que demoran los pacientes en completar las 36 sesiones se clasifican en aquellos que demoran 10 a 13 semanas y los que demoran 14 a 24 semanas. Según el tiempo transcurrido entre la cirugía y el inicio del programa se clasificaron en los que ingresan hasta la 8va semana y aquellos que ingresan entre la semana 9 y 30 . Según el tipo de cirugía a la que son sometidos se clasificaron en PAC y PTCA. Finalmente, según las sesiones realizadas se clasifican en aquellos que realizaron 12 sesiones y los que realizaron 36 .

\subsection{Programa de rehabilitación}

Se monitorizó la frecuencia cardíaca y la presión arterial basal y se programaron 12 minutos de bicicleta estática y 12 minutos de caminata en treadmill, con una carga del 50\% de los METs alcanzados en el test de esfuerzo. Se monitorizó constantemente la frecuencia cardíaca con un reloj polar y se controló la presión arterial y la sensación subjetiva de fatiga según la escala de Borg al final de cada ejercicio. El aumento de la frecuencia cardíaca de reserva, según Karvonen, no debe sobrepasar el $60 \%$ para esta carga. La frecuencia cardíaca de reserva (FCR) $=($ Frecuencia cardiaca máxima - frecuencia cardíaca de reposo) x 0,6 + frecuencia cardíaca de reposo.

Cada paciente realizó, además, 2 series de 12 repeticiones en las máquinas de isquiotibiales, mesa de cuádriceps, press pierna, press banca y pull down al $60 \%$ del $\mathrm{R}$ máximo obtenido en la evaluación inicial.

Se finalizó con 5 minutos de elongación general y se volvió a controlar la frecuencia cardíaca y la presión arterial al final de la sesión.

\subsection{Progresión}

El tiempo en el treadmill y la bicicleta estática se aumentó 2 minutos por semana hasta alcanzar los 20 minutos por máquina. La intensidad fue la necesaria para mantener un $60 \%$ de la FCR al 1er mes, $65 \%$ de la FCR al $2^{\circ}$ mes y $70 \%$ de la FCR al 3er mes.

El aumento de la duración e intensidad del ejercicio fue restringido si se observó ascenso de la presión arterial sobre 200/100 o, si en la escala subjetiva de Boher, el paciente refirió una sensación subjetiva de fatiga sobre 15 puntos.

\subsection{Análisis estadístico}

Los resultados del test de marcha fueron analizados mediante promedio y error estándar. La distribución normal se confirmó mediante la prueba de Shapiro-Wilk's y la homogeneidad de varianza mediante "robust equal va- 
riance test". La prueba " $t$ " pareada o independiente se utilizó para determinar si existía diferencia significativa entre las variables dicotómicas y el análisis de varianza en caso de variables que contuviesen más de dos grupos. La significancia estadística se estableció en la probabilidad de un error tipo I igual a 0.05. Los datos fueron ingresados a una base de datos en un programa Excel y analizados posteriormente mediante el paquete estadístico Stata 13.

\section{Resultados:}

\subsection{Efectos sobre la capacidad de marcha}

De los 760 pacientes que ingresaron al programa de rehabilitación cardiovascular entre abril de 2005 y diciembre de 2015, 67 cumplieron con los criterios para poder participar en el estudio.

Se comparó la distancia recorrida en el test de marcha de 6 minutos evaluada al inicio del PRC con la distancia recorrida en el $2^{\circ}$ test de marcha evaluada al completar las sesiones de PRC. Globalmente, los 67 pacientes mejoraron en promedio la distancia recorrida de 526.6 a 586.51 metros (promedio 112\%) (Tabla 2).

\begin{tabular}{|c|c|c|c|c|c|c|}
\hline & \multirow[t]{2}{*}{$\mathbf{N}$} & \multirow{2}{*}{$\begin{array}{l}\text { Media } \\
\text { (metros) }\end{array}$} & \multirow[t]{2}{*}{ DS } & \multicolumn{2}{|c|}{$95 \%$ IC } & \multirow[t]{2}{*}{$p$} \\
\hline & & & & Inferior & Superior & \\
\hline TM1 & 67 & 511,4 & 74,6 & 489,5 & 533,3 & \\
\hline TM2 & 67 & 573,4 & 73,6 & 551,0 & 595,8 & $<0.001$ \\
\hline
\end{tabular}

IC: intervalo de confianza; TM1: test de marcha inicial; TM2: test de marcha al final del programa; IC: intervalo de confianza al 95\%

\subsection{Incremento de la capacidad de marcha de acuerdo} a la edad

Los pacientes de diferentes grupos de edad mejoraron significativamente la distancia de marcha, sin diferencias entre los grupos. (Tabla 3)

\begin{tabular}{|c|c|c|c|c|c|}
\hline Edad (años) & $\mathrm{N}$ & $\begin{array}{c}\text { TM2-TM1 } \\
\text { (m) }\end{array}$ & DS & $\begin{array}{c}\text { TM2- TM1 } \\
\text { (\%) }\end{array}$ & $P$ \\
\hline $40-59$ & 35 & 68,8 & 57,1 & 13,5 & 0,5 \\
\hline $60-74$ & 25 & 50,7 & 39,0 & 10 & \\
\hline $75-85$ & 7 & 60.3 & 47,7 & 15,5 & \\
\hline
\end{tabular}

Abreviatura: TM2-TM1: diferencia en la distancia recorrida entre el test de marcha 2 y 1; m: metros; DS: desviación standard
3.3 Comparación del incremento del test de marcha entre los 2 tipos de revascularización

Se excluyen del análisis los 4 pacientes que presentaban tanto PTCA como cirugía de bypass. No se observó diferencias significativas en el incremento del test de marcha entre los grupos. (Tabla 4)

\begin{tabular}{|c|c|c|c|c|c|c|c|}
\hline \multirow[b]{2}{*}{ Bypass } & \multirow{2}{*}{$\begin{array}{l}N \\
46\end{array}$} & \multirow{2}{*}{$\begin{array}{l}\text { TM2- } \\
\text { TM1 } \\
(\mathrm{m}) \\
57,0\end{array}$} & \multirow{2}{*}{$\begin{array}{l}\text { DS } \\
\text { 44,5 }\end{array}$} & \multirow{2}{*}{$\begin{array}{c}\text { TM2-TM1 } \\
\text { (\%) } \\
13,4\end{array}$} & \multicolumn{2}{|c|}{$\begin{array}{l}95 \% \text { Intervalo de } \\
\text { confianza } \\
\text { (m) }\end{array}$} & \multirow{2}{*}{$\begin{array}{l}P \\
0,2\end{array}$} \\
\hline & & & & & 43,8 & 70,2 & \\
\hline PTCA & 21 & 70,3 & 60,8 & 11,5 & 42,6 & 98 & \\
\hline
\end{tabular}

3.4 Relación del incremento del test de marcha con el tiempo en que se completa el programa de rehabilitación

Los pacientes que demoraban más de 24 semanas fueron excluidos del estudio por tener menos de 1.5 sesiones por semana de promedio. Se observó que los pacientes que completaron el programa de rehabilitación dentro del período estipulado y sugerido (10 a 13 semanas) recorrieron considerablemente más metros en el test de marcha al final del programa de rehabilitación que al inicio del programa $(\mathrm{p}<0.05)($ Tabla 5)

Tabla 5. Incremento de la distancia de marcha y el tiempo que demora en completar el programa de rehabilitación

\begin{tabular}{|lccccccc} 
& $\begin{array}{l}\text { N } \\
\end{array}$ & $\begin{array}{c}\text { TM2- } \\
\text { TM1 } \\
(\mathrm{m})\end{array}$ & $\begin{array}{c}\text { DS } \\
\text { TM2-TM1 } \\
\text { (\%) }\end{array}$ & $\begin{array}{c}\text { 95\% Intervalo de } \\
\text { confianza } \\
(\mathrm{m})\end{array}$ & $\mathrm{P}$ \\
\hline 0-8 semanas & 34 & 90,5 & 45,1 & 19,5 & 78,1 & 104,9 & 0,003 \\
9-24 semanas & 21 & 55.1 & 20,5 & 10,3 & 44,9 & 65,3 & \\
\hline
\end{tabular}

Abreviatura: TM2-TM1: diferencia en la distancia recorrida entre el test de marcha 2 y 1; m: metros; DS: desviación standard

3.5 Comparación del incremento del test de marcha y el tiempo que media entre la cirugía y el programa de rehabilitación cardiovascular

Según el tiempo que transcurre entre la cirugía y el inicio del programa de rehabilitación cardiovascular, aquellos pacientes que ingresaron antes de la 8va semana post operatoria incrementaron la distancia recorrida en el test de marcha en forma similar a los que ingresaron entre la semana 9 y 24 (Tabla 6). 


$\begin{aligned} & \text { Tabla 6. Incremento de la distancia de marcha y el tiempo que } \\
& \text { media entre la cirugía y el inicio del programa de rehabilitación }\end{aligned}$
\begin{tabular}{rrrrrrrr} 
N & $\begin{array}{c}\text { TM2- } \\
\text { TM1 } \\
\text { (m) }\end{array}$ & $\begin{array}{c}\text { DS2-TM1 } \\
\text { (\%) }\end{array}$ & $\begin{array}{c}\text { 95\% Intervalo de } \\
\text { confianza } \\
\text { (m) }\end{array}$ & \\
\hline 0-8 semanas & 38 & 68,3 & 53,6 & 13,4 & 50,7 & 85,9 & 0,2 \\
9-24 semanas & 29 & 51,7 & 44,1 & 11,5 & 34,9 & 68,6 &
\end{tabular}

Abreviatura: TM2-TM1: diferencia en la distancia recorrida entre el test de marcha 2 y 1; m: metros; DS: desviación standard.

\subsection{Comparación del incremento del test de marcha y la capacidad funcional al inicio del programa de reha- bilitación cardiovascular}

La distancia recorrida en el test de marcha fue globalmente mayor en los distintos grupos de capacidad funcional (Tabla 7).

\begin{tabular}{|c|c|c|c|c|c|c|c|}
\hline \multirow[b]{2}{*}{$<5$ Mets } & \multirow{2}{*}{$\begin{array}{l}\mathrm{N} \\
11\end{array}$} & \multirow{2}{*}{$\begin{array}{c}\text { TM2- } \\
\text { TM1 } \\
(\mathrm{m}) \\
88,6\end{array}$} & \multirow{2}{*}{$\begin{array}{l}\text { DS } \\
37,4\end{array}$} & \multirow{2}{*}{$\begin{array}{c}\text { Media } \\
(\%) \\
14,4\end{array}$} & \multicolumn{2}{|c|}{$\begin{array}{l}95 \% \text { Intervalo de } \\
\text { confianza } \\
\text { (m) }\end{array}$} & \multirow{2}{*}{$\begin{array}{l}P \\
\quad 0,4\end{array}$} \\
\hline & & & & & 7,4 & 23,3 & \\
\hline Entre 5 y 8 Mets & 24 & 88,2 & 56,3 & 16,8 & 10,3 & 21,9 & \\
\hline Sobre 8 Mets & 32 & 72,9 & 32,1 & 9,1 & 6,0 & 12,1 & \\
\hline
\end{tabular}

Mets: unidad de medida del índice metabólico (cantidad de energía que consume un individuo en situación de reposo) y corresponde a 3,5 $\mathrm{ml}$ O2/kg; ES: error estándar; m: metros.

\subsection{Relación del incremento de distancia recorrida en del test de marcha y el número de sesiones a la que fue derivado el paciente}

Se observó que el grupo que realizó 36 sesiones mejoró su capacidad funcional significativamente más que el grupo que realizó 12 sesiones (Tabla 8).

\begin{tabular}{|c|c|c|c|c|c|c|}
\hline & $\mathrm{N}$ & $\begin{array}{l}\text { TM2- } \\
\text { TM1 }\end{array}$ & DS & $\begin{array}{l}\text { Media(\%) } \\
\text { (mts) }\end{array}$ & $\begin{array}{l}95 \% \text { Intervalo } \\
\text { de confianza }\end{array}$ & $P$ \\
\hline 12 sesiones & 12 & 34,4 & 20,3 & $7,5 \%$ & $15,8 \quad 53,1$ & 0,002 \\
\hline 36 sesiones & 34 & 90,5 & 45,1 & $19,5 \%$ & $78,1 \quad 104,3$ & \\
\hline
\end{tabular}

\section{Discusión:}

Los resultados muestran que en pacientes sometidos a revascularización miocárdica la rehabilitación cardiovascular logra un aumento significativo en la capacidad funcional del paciente, expresado en una mayor distancia recorrida en el test de marcha.

El aumento de capacidad de marcha fue significativo y similar en los distintos grupos de edad. Esto concuerda con los resultados de estudios anteriores 19,20 y apoya la idea de incluir en estos programas a los adultos mayores que, dadas sus limitaciones iniciales, pueden obtener mejorías que son más importantes para las actividades que realizan en su vida cotidiana.

Hubiera podido esperarse que los pacientes operados, que pueden presentar algunos síntomas que interfieren con el ejercicio, especialmente dolor relacionado a esternotomía o molestias de extremidades inferiores secundarias a la extracción de venas, pudieran estar más limitados en su respuesta a la rehabilitación. Sin embargo, el tipo de revascularización miocárdica, cirugía de bypass o angioplastía coronaria, no condicionó diferencias en la respuesta al programa de rehabilitación. Entre la intervención y la evaluación del segundo test de marcha fácilmente transcurren 4 meses, tiempo bastante largo que pueden homogenizar la mejoría de la capacidad funcional entre los grupos de pacientes. Una rehabilitación más precoz debiera conducir a una mejoría de capacidad física más rápida en pacientes operados, que experimentan una disminución de dicha capacidad como consecuencia de la inactividad y otros factores relacionados con la cirugía. Todo lo anterior sugiere que no debe retardarse el inicio de la rehabilitación una vez que el paciente se encuentra estabilizado médicamente.

Uno de los hallazgos de mayor relevancia de este estudio fue la relación del éxito del programa con el tiempo en que se desarrolla la rehabilitación. En efecto, los sujetos que pudieron completar el programa en el tiempo estipulado, entre 10 y 13 semanas (con 3 sesiones semanales promedio), lograron un beneficio significativamente mayor que aquellos que demoraron más. En estos, las interrupciones del programa que significaron una disminución a menos de 2 sesiones semanales llevan a pérdida del beneficio obtenido en las sesiones precedentes. Aquellos pacientes cuyas interrupciones eran mayores no fueron incluidos en este estudio y, probablemente, pudieran no experimentar mejoría. El diseño del estudio no contemplaba indagar sobre los motivos de interrupción, que sería interesante conocer para diseñar medidas tendientes a evitarlos.

Finalmente, al comparar la variación de la distancia recorrida en el test de marcha entre los pacientes que fueron derivados a 36 sesiones de rehabilitación, lo que es sugerido por el American College of Cardiology 21 , y aquellos que realizan sólo 12 sesiones, cumpliendo el promedio de 3 sesiones semanales, se observa que, si bien ya a las 12 sesiones existe diferencia significativa en el incremento 
de la capacidad funcional respecto al inicio del programa, el grupo de 36 sesiones obtiene aún una mejoría significativa mayor. Es probable, que, si se considerase el total de los pacientes que completan el programa de 36 sesiones, independiente de la frecuencia semanal de estas, la diferencia entre los grupos no hubiese sido considerable, pero esta hipótesis requiere un nuevo estudio.

Una fortaleza de este estudio, comparado con otros que evalúan el beneficio de la rehabilitación cardiovascular, es que se basa en una muestra bastante homogénea de un grupo seleccionado de cardiópatas que han sido sometidos a revascularización, producto de haber utilizado criterios de inclusión y exclusión exigentes. De hecho, solo 67 de los posibles 760 pacientes que iniciaron el programa cumplieron con los criterios de inclusión. A diferencia de lo que ocurre en diversos otros estudios $22-25$ el presente estudio no incluye a pacientes no cardiópatas en prevención primaria. Así mismo, el programa de rehabilitación fue riguroso, efectuado en un solo centro por un número reducido de profesionales y con una evaluación tan objetiva como era posible. Por ello, pensamos que este estudio permite una estimación más precisa del beneficio de la rehabilitación en cardiópatas coronarios revascularizados, un grupo poblacional que representa un objetivo particularmente interesante para intervenciones multidisciplinarias.

Como limitaciones, es efectivo que la muestra es altamente seleccionada y hay que tener precaución en la extrapolación de estos resultados a poblaciones más amplias. También puede dudarse de la cuantificación del efecto de la rehabilitación sobre la capacidad física a través del test de marcha, dado la influencia subjetiva que puede ejercerse sobre el paciente, sobre todo por el "deseo", tanto del paciente como del que efectúa el test, de llegar a una distancia de marcha mayor en el test final. Como proyecciones de este estudio, resultaría interesante explorar hasta qué punto la mejoría de la capacidad funcional es diferente al comparar la rehabilitación efectuada en un ambiente grupal, con pacientes altamente motivados, con aquella que pudiere obtenerse con rehabilitación efectuada con individuos que trabajan en forma aislada, como puede ser en su hogar.

Finalmente, uno de los factores cuyo valor predictivo para indicar un mayor éxito de la rehabilitación, cual es la precocidad en que se inicia el programa en relación con la intervención no resultó significativo. Pensamos que ello puede deberse a que este tiempo, intervención-rehabilitación, varió exageradamente entre los individuos y limitó las posibilidades de demostrar un efecto. Debiera evaluarse esta posibilidad, de alta proyección clínica, en un grupo acotado con tiempos intervención-rehabilitación deliberadamente controlados.

\section{Conclusión:}

Todos los pacientes, independiente de su edad, tipo de cirugía (bypass o PTCA) y capacidad funcional previa, son buenos candidatos para participar en un programa de rehabilitación. No hay razón para excluir del programa a los pacientes de edad avanzada, ya que también mejoran en forma significativa. Es altamente recomendable incorporar sobre todo a pacientes con baja capacidad funcional, ya que pequeños incrementos en la tolerancia al ejercicio determinan una importante mejoría en las actividades de la vida diaria y laboral. Se sugiere que puede ser importante incorporarlos en forma precoz al programa de rehabilitación cardiovascular.

Los dos principales factores que considerar en el éxito del programa de rehabilitación son el número de sesiones a realizar, recomendándose 36 en vez de 12 y la frecuencia promedio en que se realicen estas sesiones, siendo 3 sesiones semanales las sugeridas para obtener los mayores beneficios.

\section{Agradecimientos:}

A la Dra Mónica Acevedo, Directora del Programa de Prevención Cardiovascular, y las E.U. María José Bustamante y Francisca Márquez por su valiosa colaboración. 


\section{Referencias}

1. CORRÀ U, PIEPOLI MF, CARRÉ F, HEUSCHMANN U. Secondary prevention through cardiac rehabilitation: physical activity counselling and exercise training: key components of the position paper from the Cardiac Rehabilitation Section of the European Association of Cardiovascular Prevention and Rehabilitation. European Heart Journal, 2010;31: 1967-1974.

2. BALADY GJ, WILLIAMS MA, ADES PA, BITTNER V, COMOSS P. Core components of cardiac rehabilitation/ secondary prevention programs: 2007 update. Circulation, 2007; 115: 2675-2682.

3. LEON AS, FRANKLIN BA, COSTA F, BALADY GJ, BERRA KA. Cardiac rehabilitation and secondary prevention of coronary heart disease. Circulation, 2005; 111: 369-376.

4. SANTIBAÑEZ C, PÉREZ- TERVIC C, LÓPEZ- JIMENEZ F, CORTÉS- BERGODERI M, ARAYA MV, Burdiat. Situación actual de la rehabilitación cardíaca en Chile. Rev. Med. Chile 2012; 140: 561-568.

5. STONE J, CYR C, FRIESEN M, KENNEDY-SYMONDS $\mathrm{H}$, STENE R. Canadian guidelines for cardiac rehabilitation and atherosclerotic heart disease prevention: a summary. The Canadian journal of cardiology, 2001; 17: 3B-30B.

6. TAYLOR RS, BROWN A, EBRAHIM S, JOLLIFFE J, NOORANI H. Exercise-based rehabilitation for patients with coronary heart disease: systematic review and meta-analysis of randomized controlled trials. The American journal of medicine, 2004; 116: 682-692.

7. FLETCHER GF, ADES PA, KLIGFIELD P, ARENA R, BALADY GJ. Exercise standards for testing and training. Circulation, 2013; 128: 873-934.

8. MATSUZAWA Y, SUGIYAMA S, SUMIDA H, SUGAMURA K, NOZAKI T. Peripheral endothelial function and cardiovascular events in high-risk patients. Journal of the American Heart Association, 2013; 2: e000426.

9. GUTIÉRREZ E, FLAMMER AJ, LERMAN LO, ELÍZAGA J, LERMAN A. Endothelial dysfunction over the course of coronary artery disease. European heart journal, 2013; 34: 3175-3181.

10. SANTOS CX, NABEEBACCUS AA, SHAH AM, CAMARGO LL, FILHO SV. Endoplasmic reticulum stress and Nox-mediated reactive oxygen species signaling in the peripheral vasculature: potential role in hypertension. Antioxidants \& redox signaling, 2014; 20: 121-134.
11. SANTOS KM, CERQUEIRA NETO ML, CARVALHO VO, SANTANA FILHO VJ, SILVA JUNIOR WM. Evaluation of peripheral muscle strength of patients undergoing elective cardiac surgery: a longitudinal study. Revista Brasileira de Cirurgia Cardiovascular: Orgao Oficial da Sociedade Brasileira de Cirurgia Cardiovascular, 2014; 29: 355-9.

12. ANDERSON L, OLDRIDGE N, THOMPSON DR, ZWISLER A-D, REES K. Exercise-based cardiac rehabilitation for coronary heart disease: Cochrane systematic review and meta-analysis. Journal of the American College of Cardiology, 2016; 67: 1-12.

13. THOMAS RJ, KING M, LUI K, OLDRIDGE N, PIÑA IL. AACVPR/ACC/AHA 2007 performance measures on cardiac rehabilitation for referral to and delivery of cardiac rehabilitation/secondary prevention services: endorsed by the American college of chest physicians, American college of sports medicine, American physical therapy association, Canadian association of cardiac rehabilitation, European association for cardiovascular prevention and rehabilitation, inter-American heart foundation, national association of clinical nurse specialists, preventive cardiovascular nurses association, and the society of thoracic surgeons. Journal of the American College of Cardiology, 2007; 50): 1400-1433.

14. MENEZES AR, LAVIE CJ, MILANI RV, FORMAN DE, KING M. Cardiac rehabilitation in the United States. Progress in cardiovascular diseases, 2014; 56: 522-529.

15. ACEVEDO M, KRAMER V, BUSTAMANTE MJ, YAÑEZ F, GUIDI D, CORBALÁN R, et al. Rehabilitación cardiovascular y ejercicio en prevención secundaria. Rev. Med. Chile 2013; 141: 1307-1314.

16. WILLIAMS MA, FLEG JL, ADES PA, CHAITMAN BR, MILLER NH. Secondary prevention of coronary heart disease in the elderly (with emphasis on patients $\geq 75$ years of age). Circulation, 2002; 105: 1735-1743.

17. ANDERSON L, TAYLOR RS. Cardiac rehabilitation for people with heart disease: an overview of Cochrane systematic reviews. International journal of cardiology, 2014; 177 : 348-361.

18. PEIXOTO TC, BEGOT I, BOLZAN DW, MACHADO L, REIS MS. Early exercise-based rehabilitation improves health-related quality of life and functional capacity after acute myocardial infarction: a randomized controlled trial. Canadian Journal of Cardiology, 2015; 31: 308-313. 
19. AUSTIN J, WILLIAMS R, ROSS L, MOSELEY L, HUTCHISON S. Randomised controlled trial of cardiac rehabilitation in elderly patients with heart failure. European Journal of Heart Failure, 2005; 7: 411-417.

20. LAVIE CJ, MILANI RV. Disparate effects of improving aerobic exercise capacity and quality of life after cardiac rehabilitation in young and elderly coronary patients. Journal of Cardiopulmonary Rehabilitation and Prevention, 2000; 20: 235-240.

21. SPERTUS JA, EAGLE KA, KRUMHOLZ HM, MITCHELL KR, NORMAND S-LT. American College of Cardiology and American Heart Association methodology for the selection and creation of performance measures for quantifying the quality of cardiovascular care. Journal of the American College of Cardiology, 2005; 45: 1147-1156.

22. SADEGHI M, GARAKYARAGHI M, TAGHAVI M, KHOSRAVI M, SARRAFZADEGAN N. The impacts of cardiac rehabilitation program on exercise capacity, quality of life, and functional status of coronary artery disease patients with left ventricular dysfunction. Rehabilitation Nursing, 2015; 40: 305-309.

23. REJESKI WJ, FOY CG, BRAWLEY LR, BRUBAKER PH, FOCHT BC. Older adults in cardiac rehabilitation: a new strategy for enhancing physical function. Medicine and science in sports and exercise, 2002; 34: 1705-1713.

24. HAUER T, AUSTFORD L, STONE JA, ARENA R. Influence of Gender on the Improvement in Exercise Capacity following Cardiac Rehabilitation: 1730: Board\# 18 May 31 9: 00 AM 10: 30 AM. Medicine \& Science in Sports \& Exercise, 2007; 39: S282-S283.

25. KENNEDY MD, HAYKOWSKY M, DAUB B, VAN LOHUIZEN K, KNAPIK G. Effects of a comprehensive cardiac rehabilitation program on quality of life and exercise tolerance in women: A retrospective analysis. Current controlled trials in cardiovascular medicine, 2003; 4: 1 . 